\title{
Editorial
}

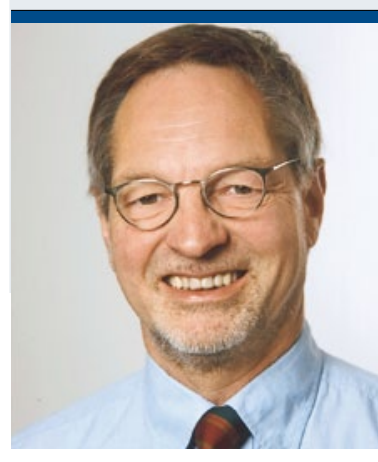

„ICh möchte nicht, dass die Urologie

wieder dahin verschwindet, wo sie herkommt."

Dr. med. Walther Grohmann

Chefredakteur und niedergelassener Urologe, München

\section{Alte Hasen und die Generation X}

D ie alten Hasen unter den Urologen erinnern sich noch: Auf dem DGU-Kongress 1987 stand auch die Wahl des BDU-Präsidenten an. Der noch amtierende Präsident Heck hielt in Erwartung seiner Wiederwahl bereits vor der anstehenden Hauptversammlung das Sektglas in der Hand, doch dann kam alles anders: Mithilfe zahlreicher Kliniker, die ebenfalls BDU-Mitglieder waren - sozusagen "Schläfer", was besser klingt als die von manchen gewählte Bezeichnung "Stimmvieh“ - wurde der damals noch junge Klaus Schalkhäuser aus dem Hut gezaubert und neuer BDU-Präsident.

Weshalb zum neuen Jahr die Erinnerung an diese alte Geschichte? Sie zeigt uns zweierlei: zum einen erkennen wir die damalige Bestrebung, die auseinanderdriftenden Verbände BDU und DGU wieder zu einer schlagkräftigen Einheit zusammenzuführen. Die vom BDU als Jahreskongress für niedergelassene Urologen veranstaltete „Urologica“ wurde danach umgehend eingestellt, ein richtiger Schritt, selbst wenn dieser Kongress die Bedürfnisse der Niedergelassenen sehr gut erfüllt hatte und die DGU-Kongresse danach einiges hiervon vermissen ließen. Und heute? Es gibt noch immer zwei urologische Hauptverbände und trotz deren Bemühung um ein einheitliches Auftreten nach Außen bleibt für alle Kooperationswilligen, Interessenten und insbesondere für die Politik die Frage bestehen, wer denn da nun eigentlich der Ansprechpartner sei. Ein gemeinsames Haus der Urologie für beide Verbände in Berlin ist zwar angedacht, aber die Realisierung steht offenbar noch in weiter Ferne. Bisher gibt es dort nur einen Raum als Platzhalter (ohne Funktion). So wünschen wir uns für das kommende Jahr, dass die Wege hierfür möglichst schnell geebnet werden.

Der zweite Gesichtspunkt zu dem alten Ereignis: Wenn das Maß voll ist, kommt manchmal alles anders. Wir wollen hiermit keineswegs zu einer Palast- revolution aufrufen. Aber wir fragen uns, weshalb sich die junge, in den Startlöchern stehende Generation X, die bald den Mittelbau der Urologie repräsentieren soll, nicht auch auf den Hauptversammlungen artikuliert. Keine Forderungen, null Ideen, tiefes Schweigen. Viele von ihnen schätzen wir als hervorragende Kollegen mit Zukunft! Sind sie in der Klinik von den DRGs erschöpft oder nur noch frustriert? Sind sie zu sehr mit der Sondierung der für sie geltenden Hackordnung beschäftigt? Und, falls niedergelassen: Bleibt ihre Vision in Anbetracht des Honorar-Frustes allein auf den kommenden EBM (der wohl auch nicht mehr Geld in den Topf bringen wird) beschränkt? Sollen es immer die wenigen Alten bleiben, die sich Sorgen um die Zukunft der Anderen machen und Alternativen für die Zukunft der Urologie aufzeigen (wie z.B. die Entwicklung der DGU hin zu einer Wissenschaftsgesellschaft), bis diese als ,ewige Kritiker" tituliert werden und kein Gehör mehr finden? Kann wirklich alles so weiterlaufen wie bisher?

Es liegt in der Hand dieser Generation X, die Zukunft der Urologie und somit ihre eigene Zukunft zu gestalten. Die Macht dazu hat sie! Den ,alten Hasen, für die schon alles gelaufen ist", könnte das egal sein. Aber wie sagte einer von denen: „Ich möchte nicht, dass die Urologie nach all dem, was sie auf den Weg gebracht hat, wieder dahin verschwindet, wo sie herkommt."

Dass dem nicht so wird, hofft auch, verbunden mit allen guten Wünschen für 2013 zusammen mit der URO-NEWS Redaktion

Ihr

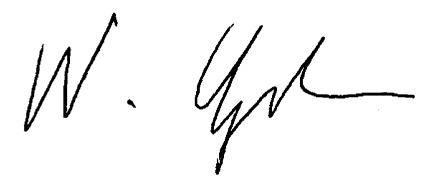

